\title{
Internal symmetric waves of two-layer fluids over an obstruction
}

\author{
H. J. Kim W. S. Bae J. W. Choi*
}

(Received 28 October 2005; revised 26 July 2006)

\begin{abstract}
We study two-dimensional capillary-gravity waves on the interface between two immiscible, inviscid and incompressible fluids of different constant densities bounded by two horizontal rigid boundaries with small obstructions with compact support. A forced modified Korteweg-de Vries equation is derived as a model equation without assuming that the fluid is of constant depth at far upstream. Various new types of steady solutions have been obtained numerically.
\end{abstract}

\section{Contents}

\section{Introduction}

*Department of Mathematics, Korea University, Seoul, 136-701, Korea. mailto:jchoi@korea.ac.kr

See http://anziamj . austms.org.au/V47EMAC2005/Choi for this article, (c) Austral. Mathematical Soc. 2006. Published August 21, 2006. ISSN 1446-8735 


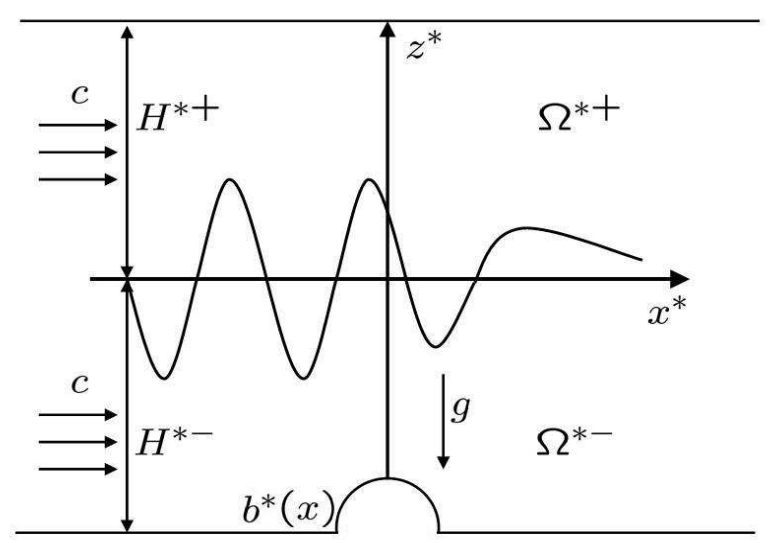

Figure 1: fluid domain.

2 Derivation of the forced modified $\mathrm{KdV}$ equation

3 Forced modified $\mathrm{KdV}$ equation

\section{Introduction}

A two-dimensional flow of immiscible, inviscid and incompressible fluids of different constant densities, bounded by two horizontal rigid boundaries is considered as in Figure 1. Let us assume that the object at the bottom is moving at a constant speed along lower boundary. By deploying a Lagrangian 
moving coordinate system, the problem is reduced to a steady state, two layer, flow past an obstruction.

Shen, Shen and Sun [1] developed an asymptotic theory for steady state, single layer, flow of small amplitude past an obstruction and derived the Forced Korteweg-de Vries equation (FKdV). Choi, Sun and Shen $[2,3]$ derived a Forced Modified K-dV equation (FMKdv) and Forced Extended K-dV equation on two-layer flows when the FKdV theory fails in two layer flows. Choi, Lim, Ahn and Park [4] also developed an asymptotic theory and derived the Forced Korteweg-de Vries equation for steady state, single layer, flow of small amplitude past an obstruction without assuming that the fluid is of constant depth far upstream. In this paper, we consider the same fluid domain as in [2] and the same type of FMKdV is derived when the density ratio is the square of depth ratio. However, we do not assume that the interface between two fluids is constant far upstream. New types of symmetric solutions of the FMKdV are found by considering periodic wave solutions for the waves ahead of an obstruction.

\section{Derivation of the forced modified $\mathrm{KdV}$ equation}

The flow under consideration in Figure 1 has following governing equations and boundary conditions [2]. In the domains $\Omega^{* \pm}$,

$$
\begin{aligned}
& u_{x^{*}}^{* \pm}+w_{z^{*}}^{* \pm}=0, \\
& u^{* \pm} u_{x^{*}}^{* \pm}+w^{* \pm} u_{z^{*}}^{* \pm}=-p_{z^{*}}^{* \pm} / \rho^{* \pm}, \\
& u^{* \pm} w_{x^{*}}^{* \pm}+w^{* \pm} w_{z^{*}}^{* \pm}=-p_{z^{*}}^{* \pm} / \rho^{* \pm}-g .
\end{aligned}
$$

At the interface $z^{*}=\eta^{*}$,

$$
u^{* \pm} \eta_{x^{*}}^{*}-w^{* \pm}=0,
$$




$$
p^{*+}-p^{*-}=T^{*} \eta_{x^{*} x^{*}}^{*} /\left(1+\left(\eta_{x^{*}}^{*}\right)^{2}\right)^{3 / 2} .
$$

At the rigid boundaries $z^{*}=H^{* \pm}\left(x^{*}\right)$,

$$
w^{* \pm}-u^{* \pm} H_{x^{*}}^{* \pm}=0
$$

where $\left(u^{* \pm}, w^{* \pm}\right)$ are velocity vectors, $\rho^{* \pm}$ are constant densities, $p^{* \pm}$ are pressures and $g$ is the gravitational acceleration.

Let $L$ be the horizontal length scale and $H$ be the vertical length scale, assuming that, far upstream, the interface of two fluids is periodic and $H$ is the mean depth of the lower fluid. Assume $\epsilon=(H / L) \ll 1$ and introduce following variables: $x=x^{*} / L, z=z^{*} / H, u^{ \pm}=u^{* \pm} / \sqrt{g H}$, $w^{ \pm}=\epsilon^{-1} w^{* \pm} / \sqrt{g H}, p^{ \pm}=p^{* \pm} / \rho^{*-} g H, \eta=\epsilon^{-1} \eta^{*} / H, T=T^{*} / \rho^{*-} g H^{2}$, $\rho^{ \pm}=\rho^{* \pm} / \rho^{*-}, h_{0}^{ \pm}=H^{* \pm} / H$. Then the above equations become nondimensionalized. Let $\rho^{+}=\rho, \rho^{-}=1, h_{0}^{+}=h^{+}=h$, and $h_{0}^{-}=h^{-}+\epsilon^{3} b(x)$ with the conditions that $h^{-}=-1$ and $b(x)$ has finite support. In addition, let $u$, $w$ and $p$ possess asymptotic expansions of the form

$$
\phi(x, z, \epsilon) \sim \phi_{0}+\epsilon \phi_{1}+\epsilon^{2} \phi_{2}+\cdots,
$$

with $u_{0}^{ \pm}=u_{0}=$ constant, $p_{0}^{ \pm}=-\rho^{ \pm} z$ and $w_{0}^{ \pm}=0$.

From the first order approximation, in $h^{-}<z<0$ and $0<z<h^{+}$,

$$
\begin{aligned}
& u_{1 x}^{ \pm}+w_{1 z}^{ \pm}=0, \\
& u_{0} u_{1 x}^{ \pm}=-p_{1 x}^{ \pm} / \rho^{ \pm}, \\
& p_{1 z}^{ \pm}=0 ;
\end{aligned}
$$

with $w_{1}^{+}=w_{1}^{-}$at $z=0$,

$$
\begin{aligned}
& p_{1}^{+}-p_{1}^{-}=-\eta\left(\rho^{+}-\rho^{-}\right) \quad \text { at } z=0, \\
& w_{1}^{ \pm}=0 \text { at } z=h^{ \pm} .
\end{aligned}
$$

Equation (3) implies that $p_{1}^{ \pm}$are functions of $x$ only. From Equations (1), (2) and (6), it follows that

$$
w_{1}^{ \pm}=\left(z-h^{ \pm}\right) p_{1 x}^{ \pm} /\left(u_{0} \rho^{ \pm}\right) .
$$


From Equations (4) and (5)

$$
p_{1 x}^{ \pm}=c_{1}^{ \pm} \eta_{x}
$$

where $c_{1}^{+}=\rho(\rho-1) /(h+\rho), \quad c_{1}^{-}=h(1-\rho) /(h+\rho)$. From Equation (2),

$$
u_{1}^{ \pm}=-\eta\left(c_{1}^{ \pm} / u_{0} \rho^{ \pm}\right)+\lambda_{1}(z)
$$

where $\lambda_{1}(z)$ are arbitrary functions of $z$. Note that we obtain $u_{1}^{ \pm}, w_{1}^{ \pm}$ and $p_{1}^{ \pm}$in terms of $\eta$. In a similar way, one can find expressions for $u_{2}^{ \pm}(x, z)$ and $w_{2}^{ \pm}(x, z)$ :

$$
\begin{aligned}
u_{2}^{ \pm}= & \frac{c_{1}^{ \pm}}{u_{0}^{2} \rho^{ \pm}}\left[\lambda_{1}(z)-\left(z-h^{ \pm}\right) \lambda_{1 z}(z)\right] \eta-\frac{1}{2} \frac{c_{1}^{ \pm 2}}{u_{0}^{3} \rho^{ \pm 2}} \eta^{2} \\
& -\frac{1}{u_{0} \rho^{ \pm}}\left(\frac{c_{2}}{2} \eta^{2}+c_{3} \eta\right)+\lambda_{2}(z) \\
w_{2}^{ \pm}= & 2\left(\frac{c_{1}^{ \pm 2}}{u_{0}^{3} \rho^{ \pm 2}}+\frac{c_{2}}{2 u_{0} \rho^{ \pm}}\right) \eta \eta_{x}\left(z-h^{ \pm}\right) \\
& +\left\{\frac{c_{3}}{u_{0} \rho^{ \pm}}\left(z-h^{ \pm}\right)-\frac{c_{1}^{ \pm}}{u_{0}^{2} \rho^{ \pm}}\left[2 \int_{h^{ \pm}}^{z} \lambda_{1}(z) d z-\left(z-h^{ \pm}\right) \lambda_{1}(z)\right]\right\} \eta_{x}
\end{aligned}
$$

where

$$
\begin{aligned}
N= & \left(\frac{h^{+}}{u_{0} \rho^{+}}-\frac{h^{-}}{u_{0} \rho^{-}}\right) \\
c_{2}= & \frac{1}{N}\left\{2\left(-\frac{c_{1}^{+}}{u_{0} \rho^{+}}+\frac{c_{1}^{-}}{u_{0} \rho^{-}}\right)-\left[\left(h^{+}\right) \frac{c_{1}^{+2}}{u_{0}^{3} \rho^{+2}}-\left(-h^{-}\right) \frac{c_{1}^{-2}}{u_{0}^{3} \rho^{-2}}\right]\right\}, \\
c_{3}= & \frac{-1}{N}\left\{\frac{c_{1}^{+}}{u_{0}^{2} \rho^{+}}\left[2 \int_{h^{+}}^{0} \lambda_{1}(z) d z+\left(h^{+}\right) \lambda_{1}(0)\right]\right. \\
& \left.\quad-\frac{c_{1}^{-}}{u_{0}^{2} \rho^{-}}\left[\left(2 \int_{h^{-}}^{0} \lambda_{1}(z) d z+\left(h^{-}\right) \lambda_{1}(0)\right)\right]\right\} .
\end{aligned}
$$

Here, $\lambda_{2}(z)$ are arbitrary functions of $z$. Derivation of the FMKdV equation requires kinematic condition only for $u_{1}^{ \pm}, w_{1}^{ \pm}, u_{2}^{ \pm}, w_{2}^{ \pm}$and $w_{3}^{ \pm}$. $w_{3}^{ \pm}$can be 
derived similarly and we omit the expression of $w_{3}^{ \pm}$. Since $\epsilon u^{ \pm} \eta_{x}-w^{ \pm}=0$ at $z=\epsilon \eta$, from the zeroth order of the asymptotic expansions of $u^{-}$and $w^{-}$, we obtain $u_{0}^{2}=h(1-\rho) /(\rho+h)$. Assuming $\rho=h^{2}$ and $\lambda_{1}(z)=0$, the following Forced Modified K-dV equation (FMKdV) is obtained from the third order expansion of the kinematic condition,

$$
2 \lambda \eta_{x}+A \eta^{2} \eta_{x}+B \eta_{x x x}=C b_{x}(x)
$$

where

$$
\begin{aligned}
2 \lambda & =\lambda_{2}(0)+\int_{-1}^{0} \lambda_{2}(z) d z+\frac{\rho}{\rho+h}\left(\frac{1}{h} \int_{h}^{0} \lambda_{2}(z) d z+\int_{-1}^{0} \lambda_{2}(z) d z\right) \\
A & =\frac{6 \rho(1-\rho)(1+h)^{2}}{u_{0}(\rho+h)^{3}} \\
B & =\frac{u_{0} h}{3(\rho+h)}\left(\frac{3 T}{u_{0}^{2}}-(1+\rho h)\right), \quad \text { and } \quad C=\left(u_{0}+1\right) .
\end{aligned}
$$

We note that if $\lambda_{1}(z) \neq 0$ and $\rho \neq h^{2}$, FKdV is derived and the same analysis as in [4] can be carried out.

\section{Forced modified $\mathrm{KdV}$ equation}

We consider the above FKdV this problem into two parts according to $\tau>$ $\tau_{0}=(1+\rho h) / 3$ and $\tau<\tau_{0}$, where $\tau=T / u_{0}{ }^{2}$.

\section{$3.1 \tau>\tau_{0}$}

Let $\tau>\tau_{0}$. We rewrite the FMKdV equation as

$$
\eta_{x x x}=-a_{1} \eta^{2} \eta_{x}+a_{2} \eta_{x}+a_{3} b_{x}
$$


where

$$
\begin{aligned}
a_{1} & =\frac{6 \rho(1-\rho)(1+h)^{2}}{h u_{0}^{2}\left(\tau-\tau_{0}\right)(\rho+h)^{2}}>0, \\
a_{2} & =\frac{-2 \lambda(\rho+h)}{h u_{0}\left(\tau-\tau_{0}\right)}, \\
a_{3} & =\frac{\left(u_{0}+1\right)(\rho+h)}{h u_{0}\left(\tau-\tau_{0}\right)}>0 .
\end{aligned}
$$

Integration of the equation in $x$ leads to

$$
\eta_{x x}=-\frac{1}{3} a_{1} \eta^{3}+a_{2} \eta+a_{3} b(x) .
$$

When $b(x)=0,(10)$ has the following periodic solution if $d>0$ [2],

$$
\eta=\xi_{0}^{1 / 2} \operatorname{cn}\left(\gamma\left(x-x_{+}\right), k\right),
$$

where $\eta\left(x_{0}\right)=\alpha, \eta_{x}\left(x_{0}\right)=\beta, d=\beta^{2}+a_{1} \alpha^{4} / 6-a_{2} \alpha^{2}, \xi_{0}=\left(3 a_{2}+\right.$ $\left.3 \sqrt{a_{2}^{2}+2 a_{2} d / 3}\right) / a_{1}, \xi_{1}=\left(3 a_{2}-3 \sqrt{a_{2}^{2}+2 a_{2} d / 3}\right) / a_{1}, \gamma=\left(a_{1}\left(\xi_{0}-\xi_{1}\right) / 6\right)^{1 / 2}$, $k^{2}=\xi_{0} /\left(\xi_{0}-\xi_{1}\right)<1, x_{0}$ is a fixed point and $x_{+}$is a phase shift. If $d \leq 0$, there is no periodic solution of (10) with $b(x)=0$. By using (11) on $\{x \mid b(x)=0\}$ and by using a matching process [2], symmetric solutions of Equation (10) can be constructed numerically when $b(x)$ is symmetric. For numerical computation, we assume that $\eta$ has a local minimum $\alpha<0$ at $x_{0}<-1$ and $b(x)=\sqrt{1-x^{2}}$ for $x \in[-1,1]$ and 0 elsewhere. $\rho$ and $h$ are fixed to $\rho=0.25$ and $h=0.5$, respectively, in the numerical studies below.

Depending on the location of the local minimum point $x_{0}$ and $\lambda$ and $\alpha$ of the wave, the wave collides with the obstruction at a different point so that $\eta$ has a different shape. When $\alpha=-2.0$ and $\tau>\tau_{0}$, symmetric steady-state solutions are obtained for $\lambda \geq-2.8284$ as in Figure 2. Let us denote the lower bound of $\lambda$ by $\lambda_{s}$. For example, $\lambda_{s}=-2.8284$ when $\alpha=-2.0$. Then, $\lambda \geq \lambda_{s}$ can be partitioned depending on the number of symmetric solutions. We found four symmetric steady state solutions on $\lambda_{s} \leq \lambda<-2.69$ and two 


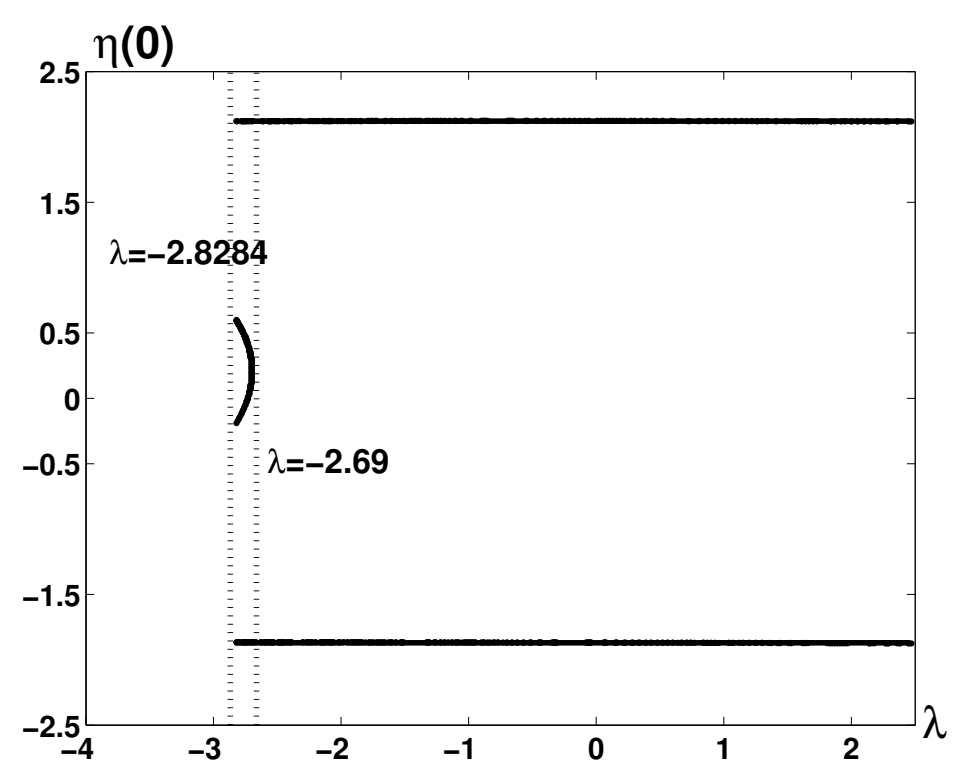

Figure 2: The relation graph between $\eta(0)$ and $\lambda$ for $\alpha=-2.0, T=3$, $\rho=0.25$ and $h=0.5$. 


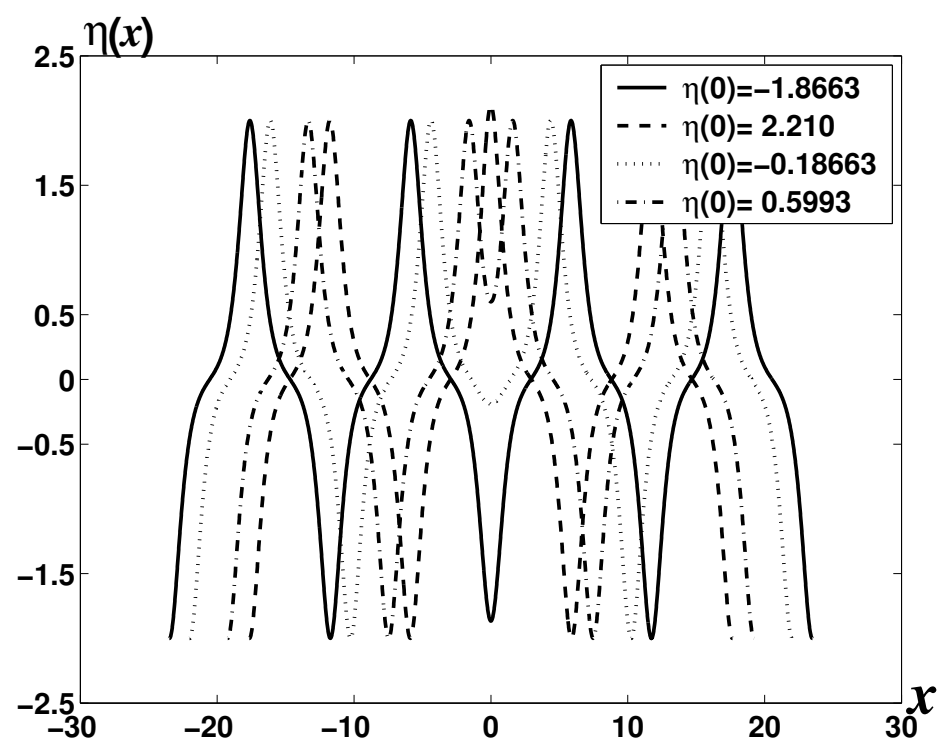

Figure 3: Four solutions when $\lambda=-2.82$ for $\alpha=-2.0, T=3, \rho=0.25$ and $h=0.5$. 


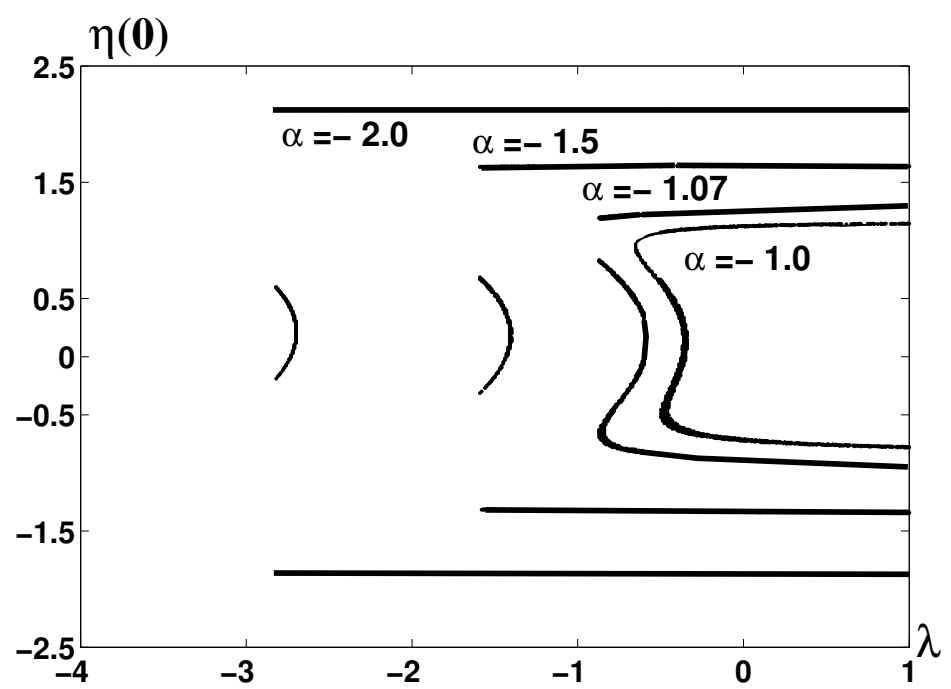

Figure 4: The relation graph between $\eta(0)$ and $\lambda$ for different $\alpha$ values with $T=3, \rho=0.25$ and $h=0.5$.

solutions on $-2.69 \leq \lambda$, respectively, when $\alpha=-2.0$. Figure 3 shows the cases when $\lambda=-2.82$.

The partition of $\lambda_{s} \leq \lambda$ with respect to the number of symmetric solutions in Figure 2 changes as the amplitude of the initial wave $|\alpha|$ varies. As in Figure 4, as $|\alpha|$ decreases, $|\eta(0)|$ correspondingly decreases and the range of $\lambda$ for symmetric solutions also decreases.

The following lower bound $\lambda_{p}$ of $\lambda$ can be derived,

$$
\lambda_{p}=-\alpha^{2} /(2 \sqrt{h(1-\rho) /(\rho+h)}) .
$$

Thus periodic solutions ahead of the bump satisfying zero mean depth are obtained for $\lambda_{p} \leq \lambda$. Since we consider symmetric solutions that are periodic ahead of the bump, $\lambda_{p} \leq \lambda_{s}$. When $\alpha$ is small, the discrepancy, $\lambda_{s}-\lambda_{p}$, is negligible. But, as $\alpha$ increases, the discrepancy increases. For example, 
for $\alpha=-2.0, \lambda_{p}=-2.828427124$ and $\lambda_{s}=-2.8284$ but for $\alpha=-0.1$, $\lambda_{p}=-0.00707107$ and $\lambda_{s}=7.11$. We anticipate that no symmetric solutions exist for $\lambda_{p} \leq \lambda<\lambda_{s}$.

\section{$3.2 \tau<\tau_{0}$}

When $\tau<\tau_{0}$, the Equation (10) is considered with $a_{1}<0$ and $a_{3}<0$. Due to different signs of the coefficients, the range of $\lambda$ for periodic solutions ahead of the bump is

$$
\lambda<\lambda_{p}=-\frac{\alpha^{2}}{\sqrt{h(1-\rho) /(\rho+h)}} .
$$

Since $\lambda_{p}<0$, we need to consider only subcritical case, $\lambda<0$. We obtained two types of solutions ahead of the bump. Let $\xi_{0}=\left(a_{2}+\sqrt{a_{2}^{2}-2 a_{1} d}\right) / a_{1}$, $\xi_{1}=\left(a_{2}-\sqrt{a_{2}^{2}-2 a_{1} d}\right) / a_{1}$ and $d$ is the same as $d$ in (11). When $\xi_{0}>\xi_{1}>0$,

$$
\eta(x)=\xi_{1}^{1 / 2} \operatorname{sn}\left(\gamma\left(x-x_{+}\right), k\right),
$$

where $\gamma=\left(a_{1} \xi_{0} / 2\right)^{1 / 2}$ and $k^{2}=\xi_{1} / \xi_{0}<1$. This solution $\eta(x)$ tends to 0 as $\xi_{1} \rightarrow 0^{+}$. When $\xi_{0}>0>\xi_{1}$,

$$
\eta(x)=\xi_{0}^{1 / 2} \operatorname{cn}\left(\gamma\left(x-x_{+}\right), k\right),
$$

where $\gamma=\left(a_{1}\left(\xi_{0}-\xi_{1}\right) / 2\right)^{1 / 2}$ and $k^{2}=\xi_{0} /\left(\xi_{0}-\xi_{1}\right)<1$. This solution $\eta(x)$ tends to $\left(2 a_{2} / a_{1}\right)^{1 / 2} \operatorname{sech}\left(a_{2}^{1 / 2} x-\phi\right)$, where $\phi$ is a phase shift determined by initial values, as $\xi_{1} \rightarrow 0^{-}$.

Figure 5 shows the relation between $\eta(0)$ and $\lambda$ from symmetric solutions when $\alpha=-0.1$ and $T=10^{-3}\left(\tau=0.002<\tau_{0}\right)$. There exist two separated regions for symmetric solutions, $-2.3903 \leq \lambda \leq-2.1484$ and $\lambda \leq-3.4227$. Periodic solutions ahead of the bump become asymmetric for $-3.4227<$ $\lambda<-2.3903$. When $\tau>\tau_{0}$, the amplitude of symmetric solutions does 


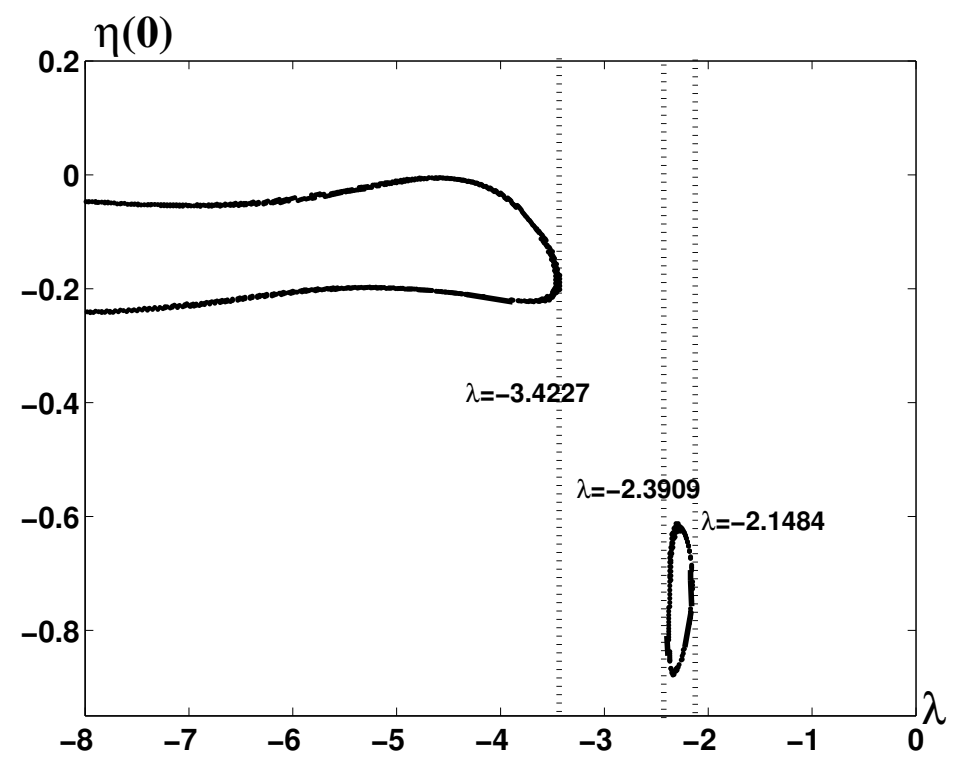

FiguRE 5: Relation graph between $\eta(0)$ and $\lambda$ for $\alpha=-0.1, T=10^{-3}$, $\rho=0.25$ and $h=0.5$. 


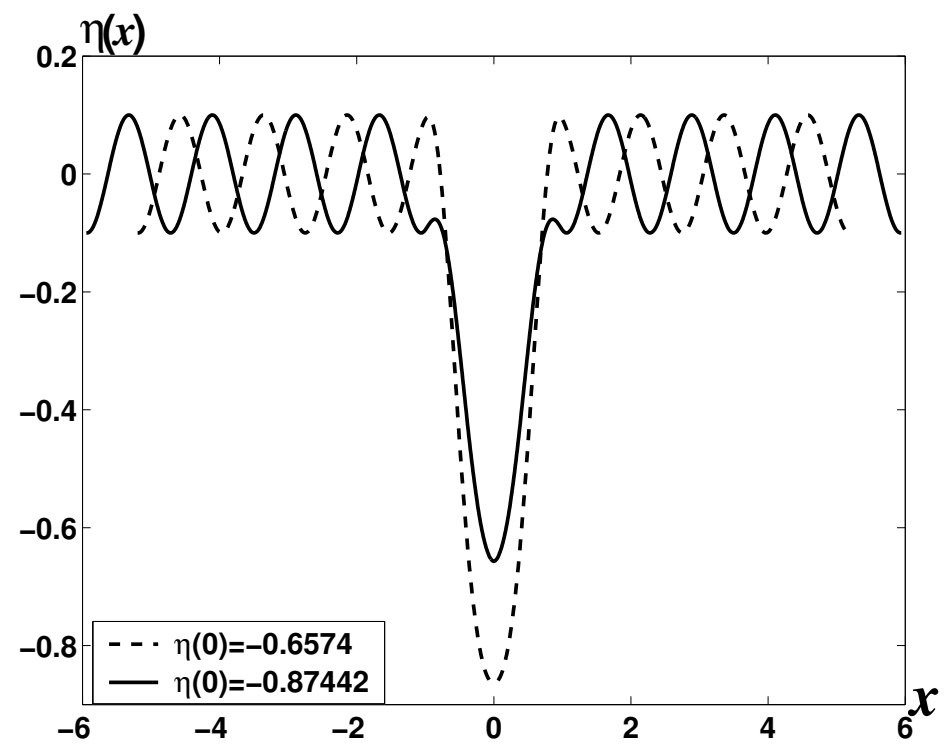

Figure 6: Two solutions when $\lambda=-2.35$ for $\alpha=-0.1, T=10^{-3}, \rho=0.25$ and $h=0.5$. 


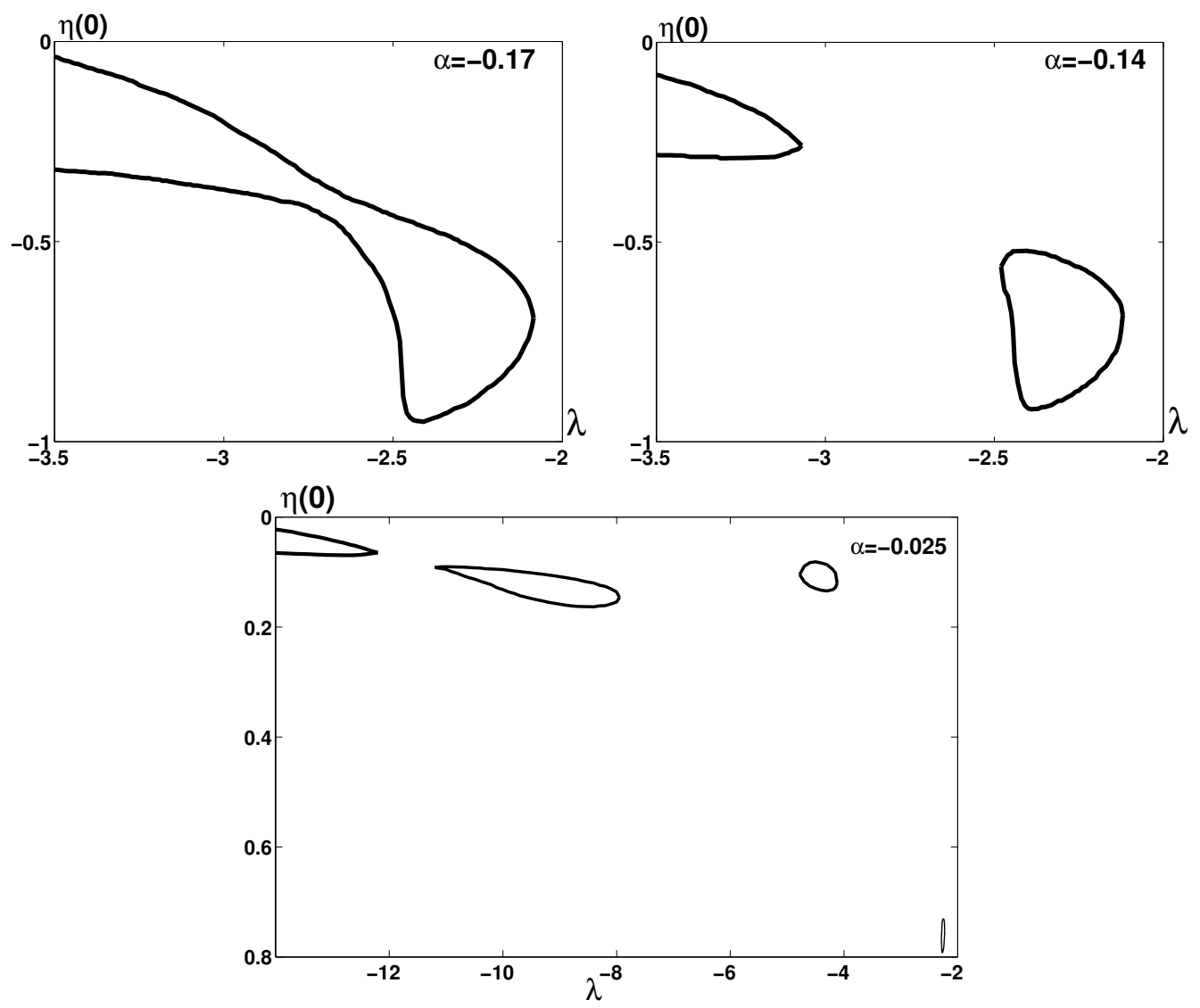

Figure 7: The graphs of $\eta(0)$ in $\lambda$ for different $\alpha$ values when $T=10^{-3}$, $\rho=0.25$ and $h=0.5$. 
not vary much from the initial perturbation $|\alpha|$ regardless of $\lambda$ (Figure 2). On the other hand, when $\tau<\tau_{0}$, the symmetric waves have large variations over obstructions (Figure 5). Figure 6 represents typical symmetric solutions when $\lambda=-2.35$.

Figure 7 shows the graphs of $\eta(0)$ in $\lambda$ for different $\alpha$ when $\tau<\tau_{0}$. When $\alpha=-0.17$, the region of $\lambda$ for symmetric solutions is connected. However, when $|\alpha|$ is decreased to $\alpha=-0.14$, these regions becomes disconnected. As $\alpha$ converges to 0 , these disconnected regions of $\lambda$ shrink to points, $\lambda=$ $-2.26,-4.46,-9.13$, This result is consistent with the symmetric solutions with crests in [2].

Let $\lambda_{s}$ be the numerical upper bound of $\lambda$ for symmetric solutions. Similarly to $\tau>\tau_{0}$, it is claimed that solutions of FMKdV will not be symmetric for $\lambda_{p} \geq \lambda>\lambda_{s}$. Two types of asymmetric solutions seem to exist. One will be an asymmetric but bounded solution, whereas the other will be an unbounded asymmetric solution.

Acknowledgments This research was partially supported by Korea University and the MIC, Korea, under the ITRC support program supervised by the IITA.

\section{References}

[1] S. P. Shen, M. C. Shen, and S. M. Sun, A model equation for steady surface waves over a bump. J. Eng. Math. 23 (1989) 315-323. http://dx.doi.org/10.1007/BF00128905 C202

[2] J. W. Choi, S. M. Sun, and M. C. Shen, Steady capillary-gravity waves on the interface of two-layer fluid over an obstruction-forced modified K-dV equation. J. Eng. Math. 28 (1994) 193-210. http://dx.doi.org/10.1007/BF00058436 C202, C206, C214 
[3] J. W. Choi, S. M. Sun, and M. C. Shen, Internal capillary-gravity waves of a two layer fluid with free surface over an obstruction-Forced Extened K-dV Equation. Phys. Fluids. A 8 (1996) 397-404. http://dx.doi.org/10.1063/1.868793 C202

[4] J. W. Choi, D. Ahn, C. H. Lim and S. Park, Symmetric surface waves over a bump. J. Korean Math. Soc. 6 (2003) 1051-1060. URL C202, C205 